\title{
YET ANOTHER NOTE ON PARACOMPACT SPACES
}

\section{E. MICHAEL 1}

1. Introduction. According to the usual definition $[1$, p. 66], a Hausdorff space $X$ is paracompact if

$\left(\mathrm{P}_{0}\right)$ Every open covering of $X$ has an open locally finite refinement. Several alternate definitions have been obtained during the past decade. Thus A. H. Stone showed [6, Theorem 1] that paracompactness is equivalent to full normality; that is, $X$ is a $T_{1}$-space, and

$\left(\mathrm{P}_{1}\right)$ Every open covering of $X$ has an open star-refinement. ${ }^{2}$ In a different direction, the author showed [4, Theorem 1$]$ that, in a regular space, $\left(\mathrm{P}_{0}\right)$ is equivalent to the following apparently weaker.

$\left(\mathrm{P}_{2}\right)$ Every open covering of $X$ has a closure-preserving ${ }^{3}$ refinement. Our principal purpose in this paper is to obtain yet another characterization of paracompactness which, in any regular space, is an easy consequence of both $\left(\mathrm{P}_{1}\right)$ and $\left(\mathrm{P}_{2}\right)$.

If $\mathcal{U}$ and $\mathcal{V}$ are collections of subsets of $X$, then we say thar $\mathcal{V}$ is cushioned in $\mathcal{u}$ if one can assign to each $V \in \mathcal{V}$ a $U_{V} \in \mathcal{U}$ such that, for every $v^{\prime} \subset \mathcal{V}$,

$$
\left(U\left\{V \mid V \in v^{\prime}\right\}\right)-\subset U\left\{U_{V} \mid V \in v^{\prime}\right\} .
$$

A refinement of $\mathcal{u}$ which is cushioned in $\mathcal{u}$ is called a cushioned refinement of $\mathcal{u}$. As examples of cushioned refinements of an open covering $\mathfrak{u}$, let us mention an open star-refinement of $\mathfrak{u}$, as well as a closure-preserving refinement of any open covering $W$ which has the property that $\{\bar{W} \mid W \in W\}$ refines $\mathcal{U}$ (such a $W$ must exist if $X$ is regular). We now have

TheOREM 1.1. A T T $T_{1}$ space $X$ is paracompact if and only if $\left(\mathrm{P}_{3}\right)$ Every open covering of $X$ has a cushioned refinement.

In $\S 2$ we obtain some properties of open coverings which are easily

Presented to the Society, January 30, 1958 under the title of Yet another characterization of paracompact spaces; received by the editors August 18, 1958.

1 Work supported by the National Science Foundation and the Office of Naval Research, contract NONR 477(21). Reproduction in whole or in part is permitted for any purpose of the United States Government.

$2 \mathcal{V}$ is a star-refinement of $\mathcal{U}$ if, for every $V_{0} \in \mathcal{V}, \cup\left\{V \in \mathcal{V} \mid V \cap V_{0} \neq \varnothing\right\}$ is a subset of some $U \in \mathcal{U}$. While $\left(P_{1}\right)$ does not follow obviously from $\left(P_{0}\right)$ in a Hausdorff space, it is nevertheless a fairly easy consequence of it; the difficult implication runs in the opposite direction.

${ }^{3} \mathcal{V}$ is closure-preserving if, for every $\mathcal{V}^{\prime} \subset \mathcal{V},\left(\cup\left\{V \mid V \in \mathcal{U}^{\prime}\right\}\right)^{-}=\cup\{\bar{V} \mid V \in \mathcal{U}\}$. Any locally finite $\mathcal{U}$ is clearly closure-preserving. 
equivalent to the property of having a cushioned refinement; one of these equivalences sheds some further light on the extent to which $\left(\mathrm{P}_{3}\right)$ is apparently weaker than full normality. The proof of Theorem 1.1 , which closely parallels that of $[4$, Theorem 1$]$, is found in $\S 3$. In $\S 4$ we use Theorem 1.1 to prove the following result, which generalizes [4, Theorem 2]; we call $\vartheta$ a $\sigma$-cushioned refinement of $\mathcal{U}$ if $\vartheta=\bigcup_{i=1}^{\infty} V_{i}$, with each $\vartheta_{i}$ cushioned in $\mathcal{u}$.

Theorem 1.2. A $T_{1}$-space $X$ is paracompact if and only if

$\left(\mathrm{P}_{3}^{\prime}\right)$ Every open covering of $X$ has an open $\sigma$-cushioned refinement.

In $\S 5$, finally, we show how Theorem 1.2 provides a simplified proof of a beautiful metrization theorem recently obtained by J. Nagata [5, Theorem 1].

2. Some equivalent properties. In the following proposition, a set $V \subset X \times X$ is called a semi-neighborhood of the diagonal if both $V(x)$ and $^{4} V^{-1}(x)$ are neighborhoods of $x$ for every $x \in X$.

Proposition 2.1. The following properties of an open covering $u$ of a topological space $X$ are equivalent.

(a) $u$ has a cushioned refinement $\mathcal{V}$.

(b) There exists an indexed covering $\left\{W_{U} \mid U \in \mathcal{U}\right\}$ of $X$ such that $\left(U\left\{W_{U} \mid U \in \mathcal{u}^{\prime}\right\}\right)-\subset U\left\{U \mid U \in \mathcal{u}^{\prime}\right\}$ for every $\mathfrak{u}^{\prime} \subset \mathcal{C}$.

(c) One can assign to each $x \in X$ a $U_{x} \in \mathcal{U}$ such that, for every $X^{\prime} \subset X$, we have $\bar{X}^{\prime} \subset \cup\left\{U_{x} \mid x \in X^{\prime}\right\}$.

(d) There exists a semi-neighborhood $V$ of the diagonal such that $\{V(x) \mid x \in X\}$ refines $u$.

Proof. It suffices to prove the following implications:

(a) $\rightarrow$ (b). Let $W_{U}=U\left\{V \in \mathcal{U} \mid U_{V}=U\right\}$.

(b) $\rightarrow$ (c). Pick $U_{x}$ such that $x \in W_{U_{x}}$.

(c) $\rightarrow$ (a). Let $V=\{\{x\} \mid x \in X\}$, and let $U_{\{x\}}=U_{x}$.

(c) $\rightarrow$ (d). Let $V=\left\{(x, y) \in X \times X \mid y \in U_{x}\right\}$. Then $V(x)=U_{x}$, which is a neighborhood of $x$. Moreover, if we let $R_{x}=\left\{y \in X \mid x \in U_{y}\right\}$, then by assumption $x \in\left(X-\bar{R}_{x}\right) \subset\left(X-R_{x}\right)=\left\{y \in X \mid x \in U_{y}\right\}=V^{-1}(x)$, and hence $V^{-1}(x)$ is also a neighborhood of $x$.

(d) $\rightarrow$ (c). Pick $U_{x} \in \mathcal{U}$ such that $V(x) \subset U_{x}$. If $X^{\prime} \subset X$ and $y \in \bar{X}^{\prime}$, then $V^{-1}(y)$ intersects $X^{\prime}$, and hence, for some $x \in X^{\prime}, x \in V^{-1}(y)$ and thus $y \in V(x)$. This completes the proof.

In conclusion, let us recall that J. L. Kelley $[2$, p. 155] calls an open covering even if it satisfies $2.1(\mathrm{~d})$ with "semi-neighborhood" replaced by "neighborhood." Since Kelley showed [2, p. 170, $U$ ] that

\footnotetext{
4 As usual, $V(x)=\{y \in X \mid(x, y) \in V\}$ and $V^{-1}(x)=\{y \in X \mid(y, x) \in V\}$.
} 
the requirement that every open covering be even is easily equivalent to full normality, it follows from Proposition 2.1 that, roughly speaking, $\left(\mathrm{P}_{3}\right)$ is related to full normality as semi-neighborhoods of the diagonal are related to neighborhoods.

3. Proof of Theorem 1.1. To prove the nontrivial part of the theorem, we assume that the $T_{1}$-space $X$ satisfies condition $\left(\mathrm{P}_{3}\right)$ in the statement of Theorem 1.1, and will prove that $X$ is paracompact. Since we shall be dealing with indexed coverings, let us make the convention that an indexed covering $\left\{C_{\alpha}\right\}_{\alpha \in \mathrm{A}}$ is a cushioned refinement of an indexed covering $\left\{U_{\alpha}\right\}_{\alpha \in \mathrm{A}}$ if, for every $\mathrm{A}^{\prime} \subset \mathrm{A}$,

$$
\left(\bigcup_{\alpha \in \mathbf{A}^{\prime}} C_{\alpha}\right)^{-} \subset \bigcup_{\alpha \in \mathbf{A}^{\prime}} U_{\alpha}
$$

As an immediate consequence of Proposition 2.1, (a) $\rightarrow$ (b), we now have

Lemma 3.1. Every indexed open covering $\left\{U_{\alpha}\right\}_{\alpha \in \mathrm{A}}$ of $X$ has an indexed cushioned refinement $\left\{C_{\alpha}\right\}_{\alpha \in \mathbf{A}}$.

Using Lemma 3.1, we next prove

Lemma 3.2. $X$ is normal.

Proof. Let $E_{1}, E_{2}$ be disjoint, closed subsets of $X$. Then $\left\{X-E_{1}\right.$, $\left.X-E_{2}\right\}$ is an open covering of $X$, so by Lemma 3.1 there exists a covering $\left\{C_{1}, C_{2}\right\}$ of $X$ such that $\bar{C}_{i} \subset X-E_{i}$ for $i=1,2$. But then the open sets $X-\bar{C}_{1}$ and $X-\bar{C}_{2}$ separate $E_{1}$ and $E_{2}$, and the proof is complete.

Since Lemma 3.2 implies that $X$ is regular, we can now prove that $X$ is paracompact by showing that every open covering of $X$ has an open $\sigma$-discrete ${ }^{5}$ refinement [4, Proposition 1].

After these preliminaries, let $\left\{U_{\alpha}\right\}_{\alpha \in \mathrm{A}}$ be an open covering of $X$, which has been indexed by a well-ordered index set $\mathbf{A}$. We must show that this covering has a $\sigma$-discrete open refinement. ${ }^{5}$

Lемма 3.3. For each positive integer $i$, there exists a cushioned refinement $\left\{C_{\alpha, i}\right\}_{\alpha \in \mathrm{A}}$ of $\left\{U_{\alpha}\right\}_{\alpha \in \mathrm{A}}$ such that, for all $\alpha$ and $i$,

(a) $\left(\mathrm{U}_{\beta<\alpha} C_{\beta, i}\right)-\cap C_{\alpha, i+1}=\varnothing$,

(b) $C_{\alpha, i} \cap\left(\cup_{\beta>\alpha} C_{\beta, i+1}\right)^{-}=\varnothing$.

Proof. Let $\left\{C_{\alpha, 1}\right\}_{\alpha \in \mathrm{A}}$ be any cushioned refinement of $\left\{U_{\alpha}\right\}_{\alpha \in \mathrm{A}}$

$5 \mathscr{W}$ is discrete if every $x \in X$ has a neighborhood intersecting at most one $W \in W$; $W$ is $\sigma$-discrete if $W=U_{i=1}^{\infty} W_{i}$, with each $W_{i}$ discrete. 
(Lemma 3.1). Suppose that suitable refinements $\left\{C_{\alpha, i}\right\}_{\alpha \in \mathrm{A}}$ have been found for $i=1, \cdots, n$, and let us construct $\left\{C_{\alpha, n+1}\right\}_{\alpha \in A}$. For all $\alpha$, let

$$
U_{\alpha, n+1}=U_{\alpha}-\left(\bigcup_{\beta<\alpha} C_{\beta, n}\right)^{-}
$$

Then $\left\{U_{\alpha, n+1}\right\}_{\alpha \in \mathrm{A}}$ is an (open) covering of $X$, because $x \in X$ implies $x \in U_{\alpha, n+1}$ for the first $\alpha$ for which $x \in U_{\alpha}$ (since, by assumption, $\left.\left(\bigcup_{\beta<\alpha} C_{\beta, n}\right)-\subset \bigcup_{\beta<\alpha} U_{\beta}\right)$. We now use Lemma 3.1 to pick a cushioned refinement $\left\{C_{\alpha, n+1}\right\}_{\alpha \in \mathrm{A}}$ of $\left\{U_{\alpha, n+1}\right\}_{\alpha \in \mathrm{A}}$. Then (a) follows at once from (1) and the fact that $C_{\alpha, n+1} \subset U_{\alpha, n+1}$. To see (b), note that, by (1), $C_{\alpha, n}$ is disjoint from $U_{\beta, n+1}$ for all $\beta>\alpha$, and hence from $\left(\bigcup_{\beta>\alpha} C_{\beta, n+1}\right)^{-}$ $\subset U_{\beta>\alpha} U_{\beta, n+1}$.

Lemma 3.4. There exists an indexed open covering

$$
\left\{V_{\alpha, i} \mid \alpha \in A, i=1,2, \cdots\right\}
$$

of $X$ such that, for all $i$,

(a) $V_{\alpha, i} \subset U_{\alpha}$ for all $\alpha$,

(b) $V_{\alpha, i} \cap V_{\beta, i}=\varnothing$ whenever $\alpha \neq \beta$.

Proof. For each $\alpha$ and $i$, let

$$
V_{\alpha, i}=X-\left(\bigcup_{\beta \neq \alpha} C_{\beta, i}\right)^{-}
$$

Since $\left\{C_{\alpha, i}\right\}_{\alpha \in \mathrm{A}}$ is a covering of $X$ for each $i$, we have

$$
V_{\alpha, i} \subset C_{\alpha, i} \subset U_{\alpha}
$$

for all $\alpha$ and $i$, which proves (a) and (b). Since each $V_{\alpha, i}$ is clearly open, it remains to show that the sets $V_{\alpha, i}$ cover $X$.

Pick an $x \in X$, and let us find a $V_{\alpha, i}$ containing it. Using the wellordering of the index set $\mathbf{A}$, let

$$
\alpha_{i}=\min \left\{\alpha \in \mathrm{A} \mid x \in C_{\alpha, i}\right\} \quad i=1,2, \cdots,
$$

and then pick a positive integer $k$ such that

$$
\alpha_{k}=\min \left\{\alpha_{i} \mid i=1,2, \cdots\right\} .
$$

Let us show that

$$
x \in V_{\alpha_{k}, k+1} .
$$

Note first that $x \in C_{\alpha_{k}, k}$ by definition of $\alpha_{k}$, and hence, by 3.3(b) (with $i=k$ ),

$$
x \notin\left(\bigcup_{\alpha>\alpha_{k}} C_{\alpha, k+1}\right)^{-} .
$$


Again from the definition of $\alpha_{k}$, we have $x \in C_{\alpha, k+2}$ for some $\alpha \geqq \alpha_{k}$, and hence

$$
x \notin\left(\bigcup_{\beta<\alpha_{k}} C_{\beta, k+1}\right)^{-}
$$

by 3.3(a) with $i=k+1$. It follows from (2) and (3) that $x \in V_{\alpha_{k}, k+1}$, which completes the proof of the lemma.

To complete the proof of Theorem 1.1, we apply Lemma 3.1 once more to obtain a cushioned refinement $\left\{D_{\alpha, i} \mid \alpha \in \mathrm{A}, i=1,2, \cdots\right\}$ of $\left\{V_{\alpha, i} \mid \alpha \in \mathrm{A}, i=1,2, \cdots\right\}$. Now for each $i,\left(\bigcup_{\alpha \in \mathbf{A}} D_{\alpha, i}\right)-\subset \bigcup_{\alpha \in \mathbf{A}} V_{\alpha, i}$ and hence, remembering that $X$ is normal (Lemma 3.1), there exists an open $G_{i} \subset X$ such that $\left(\bigcup_{\alpha \in \mathrm{A}} D_{\alpha, i}\right)-\subset G_{i} \subset \bar{G}_{i} \subset \bigcup_{\alpha \in \mathbf{A}} V_{\alpha, i}$. Letting

$$
\mathscr{W}_{i}=\left\{V_{\alpha, i} \cap G_{i} \mid \alpha \in \mathrm{A}\right\} \quad i=1,2, \cdots,
$$

we see that each $W_{i}$ is a discrete family of open sets, and that $\bigcup_{i=1}^{\infty} W_{i}$ is the required $\sigma$-discrete open refinement of $\left\{U_{\alpha}\right\}_{\alpha \in A}$.

4. Proof of Theorem 1.2. Let us begin by noting that 2.1(c) can be rephrased as follows:

$2.1\left(c^{\prime}\right)$. One can assign to each $x \in X$ a $U_{x} \in \mathcal{U}$ containing $x$, and to each $y \in X$ a neighborhood $W_{y}$ of $y$, such that $y \notin U_{x}$ implies $x \notin W_{y}$.

Suppose now that the open covering $u$ of $X$ has a $\sigma$-cushioned refinement $\mathcal{V}=\bigcup_{n=1}^{\infty} \mathcal{V}_{n}$ (with the $U \in \mathcal{U}$ assigned to a $V \in \mathcal{V}_{n}$ denoted by $\left.U_{V, n}\right)$, and let us show that $u$ satisfies $2.1\left(\mathrm{c}^{\prime}\right)$. For each $x \in X$, let $n(x)=\inf \left\{n \mid x \in U v_{n}\right\}$, pick a $V(x) \in \mho_{n(x)}$ which contains $x$, and let $U_{x}=U_{V(x), n(x)}$. For $y \in Y$, pick any index $m(y)$ such that $y \in U \mho_{m(y)}$, and let

$$
W_{\nu}=U v_{m(y)}-\bigcup_{k=1}^{m(y)}\left(U\left\{V \in v_{k} \mid y \notin U_{V, k}\right\}\right) ;
$$

then $W_{\nu}$ is a neighborhood of $y$, because each $v_{k}$ is cushioned in $\boldsymbol{u}$. To see that $2.1\left(\mathrm{c}^{\prime}\right)$ is satisfied, suppose that $y \notin U_{x}$. If $n(x) \leqq m(y)$, then $V(x)$ has been subtracted out from $W_{y}$, and hence $x \notin W_{\mathbf{y}}$. If $n(x)>m(y)$, then $x \in \cup \mho_{m(y)}$ by definition of $n(x)$, and again $x \notin W_{y}$. This completes the proof.

The above proof shows that, in Theorem 1.2, the condition that the $\sigma$-cushioned refinement $V=\bigcup_{n=1}^{\infty} \vartheta_{n}$ be open can be weakened to requiring only that the interiors of the sets $\bigcup_{V_{n}}(n=1,2, \cdots)$ cover $X$; one thus obtains a result which simultaneously generalizes Theorems 1.1 and 1.2. Going a step further, it is even sufficient to require only that, for each $y \in Y$, the set

$$
W_{y}=\bigcup_{m=1}^{\infty}\left(U \mho_{m}-\bigcup_{k=1}^{m}\left(U\left\{V \in V_{k} \mid y \notin U_{V, k}\right\}\right)\right)
$$


is a neighborhood of $y$. This last and (so far) weakest characterization of paracompactness will be applied elsewhere.

5. An application of Theorem 1.2. The following metrization theorem was recently proved by J. Nagata [5].

Theorem 5.1 (J. Nagata). For a $T_{1}$-space $X$ to be metrizable, it is necessary and sufficient that every $x \in X$ have (not necessarily open) neighborhoods $S_{n}(x)$ and $U_{n}(x)(n=1,2, \cdots)$, with $\left\{U_{n}(x)\right\}_{n=1}^{\infty} a$ local base at $x$, such that

(a) $y \in U_{n}(x)$ implies $S_{n}(y) \cap S_{n}(x)=\varnothing$.

(b) $y \in S_{n}(x)$ implies $S_{n}(y) \subset U_{n}(x)$.

Necessity is obvious; the main step in Nagata's proof of sufficiency is to show (without using (b)) that $X$ must be paracompact, after which metrizability follows easily from previously known metrization theorems. Nagata's proof of paracompactness employs an ingenious and intricate transfinite construction; our purpose in this section is to use Theorem 1.2 to give a very simple proof (also without assuming (b)).

Let $W$ be an open covering of $X$. For each $n$, let ${ }^{6}$

$$
v_{n}=\left\{S_{n}^{0}(x) \mid U_{n}(x) \subset W \text { for some } W \in W\right\},
$$

and let $v=\bigcup_{n=1}^{\infty} \vartheta_{n}$. Then $v$ is an open covering of $X$, and it only remains to check that each $\mathcal{V}_{n}$ is cushioned in $W$. For each $V \in \mathcal{V}_{n}$, pick a $W_{V} \in W$ such that, for some $x \in X, V=S_{n}^{0}(x)$ and $U_{n}(x) \subset W_{V}$. To see that this works, let $\mathcal{V}^{\prime} \subset \mathcal{V}_{n}$, and let $y \in U\left\{W_{V} \mid V \in \mathcal{V}^{\prime}\right\}$; it then follows from (a) that $S_{n}(y) \cap V=\varnothing$ for all $V \in \mathcal{V}^{\prime}$, and hence $y \notin\left(\cup\left\{V \mid V \in U^{\prime}\right\}\right)^{-}$. This completes the proof.

\section{REFERENCES}

1. J. Dieudonné, Une généralization des espaces compacts, J. Math. Pures Appl. vol. 23 (1944) pp. 65-76.

2. J. L. Kelley, General topology, New York, Van Nostrand, 1955.

3. E. Michael, $A$ note on paracompact spaces, Proc. Amer. Math. Soc. vol. 4 (1953) pp. 831-838.

4. Another note on paracompact spaces, Proc. Amer. Math. Soc. vol. 8 (1957) pp. 822-828.

5. J. Nagata, A theorem for metrizability of a topological space, Proc. Japan Acad. vol. 33 (1957) pp. 128-130.

6. A. H. Stone, Paracompactness and product spaces, Bull. Amer. Math. Soc. vol. 54 (1948) pp. 977-982.

\section{UNIVERSITY OF WASHINGTON}

6 $S_{n}^{0}(x)$ will denote the interior of $S_{n}(x)$. 